\title{
Phosphorylation of Puma modulates its apoptotic function by regulating protein stability
}

\author{
M Fricker ${ }^{\star, 1,2}$, J O'Prey ${ }^{1}$, AM Tolkovsky ${ }^{2,3}$ and KM Ryan ${ }^{\star, 1}$
}

Puma is a potent BH3-only protein that antagonises anti-apoptotic Bcl-2 proteins, promotes Bax/Bak activation and has an essential role in multiple apoptotic models. Puma expression is normally kept very low, but can be induced by several transcription factors including p53, p73, E2F1 and FOXO3a, whereby it can induce an apoptotic response. As Puma can to bind and inactivate all anti-apoptotic members of the Bcl-2 family, its activity must be tightly controlled. We report here, for the first time, evidence that Puma is subject to post-translational control through phosphorylation. We show that Puma is phosphorylated at multiple sites, with the major site of phosphorylation being serine 10. Replacing serine 10 with alanine causes reduced Puma turnover and enhanced cell death. Interestingly, Puma turnover occurs through the proteasome, and substitution of serine 10 causes elevated Puma levels independently of macroautophagy, Bcl-2 family member binding, caspase activity and apoptotic death. We conclude, therefore, that phosphorylation of Puma at serine 10 promotes Puma turnover, represses Puma's cell death potential and promotes cell survival. Owing to the highly pro-apoptotic nature of Puma, these studies highlight an important additional regulatory step in the determination of cellular life or death.

Cell Death and Disease (2010) 1, e59; doi:10.1038/cddis.2010.38; published online 29 July 2010

Subject Category: Cancer

The Bcl-2 family proteins have a crucial role in determining cell fate by regulating the release of apoptogenic factors from mitochondria through a process known as mitochondrial outer membrane permeabilisation (MOMP). ${ }^{1,2} \mathrm{Bcl}-2$ proteins are divided into three sub-groups, on the basis of function and the number of $\mathrm{Bcl}$ homology $(\mathrm{BH})$ domains they possess. ${ }^{1,2}$ The anti-apoptotic $\mathrm{Bcl}-2$ family proteins, including $\mathrm{Bcl}-2$, $\mathrm{Bcl}-\mathrm{xL}$ and $\mathrm{Mcl}-1$, typically contain 3-4 BH domains, which form a groove on the protein surface through which pro-apoptotic Bcl-2 family proteins are bound. The pro-apoptotic $\mathrm{Bcl}-2$ family proteins are classified into two sub-groups, the multi-domain proteins Bax and Bak, which contain $\mathrm{BH}$ domains $1-3$, and the $\mathrm{BH} 3$-only proteins, which, as the name suggests, possess only the $\mathrm{BH} 3$ domain. ${ }^{3}$ On receiving an apoptotic stimulus, Bax and Bak homo-oligomerise on the outer mitochondrial membrane, leading to MOMP and subsequent caspase activation. ${ }^{4}$ In non-apoptotic cells, monomeric Bax and Bak are held in check through inhibitory interactions with anti-apoptotic Bcl-2 proteins. ${ }^{1,2,5} \mathrm{BH} 3$-only proteins contribute to the induction of apoptosis by competing with and displacing Bax and Bak from their inhibitory interactions with anti-apoptotic $\mathrm{Bcl}-2$ proteins. ${ }^{3,6}$ In addition, a subset of $\mathrm{BH} 3-$ only proteins can directly interact with and activate Bax/Bak. ${ }^{7-9}$ In the absence of Bax and Bak, the binding of $\mathrm{BH} 3-o n l y$ proteins to anti-apoptotic $\mathrm{Bcl}-2$-binding partners is insufficient to induce apoptosis. ${ }^{3,10}$
The BH3-only proteins are the largest subgroup of the Bcl-2 family, comprising at least 12 members: Bad, Bim, Bik/Nbk/Blk, Bid, Bmf, Puma, Noxa, Spike, BNIP3, Nix, Hrk/DP5 and Beclin-1. ${ }^{3}$ Given their integral role in determining cell fate, it is not surprising that the activity of BH3-only proteins is stringently controlled, both through transcriptional regulation and post-translational mechanisms such as phosphorylation. ${ }^{11}$ Puma was originally identified during large-scale analysis for novel p53-inducible genes and Bcl-2-interacting proteins. ${ }^{12-14}$ Puma is an extremely potent $\mathrm{BH} 3-$ only protein, having the capacity to bind with high affinity to all known anti-apoptotic $\mathrm{Bcl}-2$ proteins. ${ }^{15}$ In addition, Puma may also directly interact with and activate Bax/Bak, for example, through an interaction with the first $\alpha$-helix of Bax. ${ }^{7,8}$ In keeping with its highly pro-apoptotic nature, Puma has been shown to have an essential role in the induction of apoptosis in numerous in vitro and in vivo systems and cell types including neurons, lymphocytes, thymocytes, fibroblasts and cardiomyocytes. ${ }^{15,16}$ Puma expression is regulated by transcription, and factors regulating Puma transcription include p53, p73, E2F and FOXO3a. ${ }^{12,13,17-19}$ To date, however, no studies have described the regulation of Puma activity by post-translational means, despite numerous papers describing the posttranslational regulation of other $\mathrm{BH} 3-$ only proteins including Bad, Bim, Bmf and Bik. ${ }^{20-24}$

\footnotetext{
${ }^{1}$ Tumour Cell Death Laboratory, Beatson Institute for Cancer Research, Garscube Estate, Switchback Road, Glasgow G61 1BD, UK; ${ }^{2}$ Department of Biochemistry, University of Cambridge, Cambridge CB2 1QW, UK and ${ }^{3}$ Cambridge Centre for Brain Repair, University of Cambridge, Cambridge CB2 OPY, UK

*Corresponding author: KM Ryan, Beatson Institute for Cancer Research, Garscube Estate, Switchback Road, Glasgow G61 1BD, UK. Tel: + 441413303655 ;

Fax: + 4401419426 521; E-mail: k.ryan@ beatson.gla.ac.uk or M Fricker, Department of Biochemistry, University of Cambridge, Cambridge CB2 1QW, UK.

Tel: + 44122333 3701; Fax: + 44122333 3345; E-mail: mf309@ cam.ac.uk

Keywords: Puma; phosphorylation; protein stability

Abbreviations: Baf, Boc-aspartyl-(O-methyl)-fluoromethylketone; BH, Bcl homology; DKO MEF, Bax/Bak-null mouse embryo fibroblast; MAPK, mitogen-activated protein kinase; MOMP, mitochondrial outer membrane permeabilisation; 1D-TLC, one-dimensional thin-layer chromatography

Received 10.6.10; accepted 15.6.10; Edited by V De Laurenzi
} 
Here, we provide the first demonstration that Puma is subject to post-translational regulation by phosphorylation. We find that Puma is phosphorylated on several evolutionarily conserved residues, with the principal site of phosphorylation being Serine 10. An inability to phosphorylate this site results in decreased Puma turnover, leading to increased apoptotic death. Given the critical role of Puma downstream of p53 and in multiple additional cell death scenarios, these studies, therefore, highlight another potentially critical control point in the cellular decision between life and death.

\section{Results}

Exogenous Puma- $\alpha$ is phosphorylated in HeLa cells on serine residues. To investigate whether Puma might be phosphorylated, we transiently transfected HeLa cells with constructs encoding $\mathrm{N}$-terminally $\mathrm{HA}$ - or Flag-tagged Puma for the purpose of labelling studies. In the absence of caspase inhibitors, HeLa cells underwent apoptosis in as little as $4 \mathrm{~h}$ after transfection because of Puma expression (data not shown), leading to an insufficient yield of Puma protein for immunoprecipitation (IP). However, after expression of Puma in HeLa cells for 12-18 h in the presence of the pan-caspase inhibitor Boc-aspartyl-(O-methyl)-fluoromethylketone (BAF), both $\mathrm{N}$-terminally $\mathrm{HA}$ - and Flag-tagged Puma were successfully immunoprecipitated (Figure 1a and c). To ascertain whether Puma was phosphorylated in HeLa cells, at $12-16 \mathrm{~h}$ after transfection, HeLa cells were labelled with ${ }^{32} \mathrm{P}$-orthophosphate before lysis and IP. Interestingly, IP of both $\mathrm{N}$-terminal Flag- and HA-tagged Puma resulted in the detection of an identical ${ }^{32} \mathrm{P}$-labelled doublet, indicating that the phosphorylation of the proteins was not in any way determined by either the HA- or FLAG tag (Figure $1 \mathrm{~b}$ and $\mathrm{c}$ ). A non-apoptotic HA-tagged Puma construct in which the $\mathrm{BH} 3$ domain was deleted $(\triangle \mathrm{BH} 3)$ was also phospholabelled when transfected into HeLa cells (Figure 1b). More radiolabel appeared to be incorporated into this mutant, indicating not only that the $\mathrm{BH} 3$ domain is dispensable for phosphorylation of Puma but also that regions within the $\mathrm{BH} 3$ domain may potentially repress phosphorylation elsewhere in the protein. Although this specific possibility is yet to be determined, these results clearly show that exogenous Puma is phosphorylated in untreated HeLa cells in a BH3-domain-independent manner.

To determine the type(s) of residues in Puma that are phosphorylated, phosphoamino acid analysis by onedimensional thin-layered chromatography (1D-TLC) was used to identify whether serine, threonine or tyrosine residues were phosphorylated in ${ }^{32} \mathrm{P}$-labelled cells expressing $\mathrm{HA}-\mathrm{Puma}-\Delta \mathrm{BH} 3$. Phosphoserine, phosphothreonine and phosphotyrosine standards were separated by 1D-TLC and detected by ninhydrin staining (Figure 1d and Supplementary Figure 1). When ${ }^{32} \mathrm{P}$-labelled HA-Puma amino acids were run on a TLC plate with phosphoamino acid standards, a strong ${ }^{32} \mathrm{P}$ signal was observed overlying the phosphoserine standard, indicating that serine, but not threonine or tyrosine residues, are phosphorylated in HA-Puma (Figure 1d).
Serine $\mathbf{1 0}$ is the major site of phosphorylation in Puma- $\alpha$. Examination of the human Puma- $\alpha$ sequence shows 11 possible serines that could be sites of phosphorylation (Figure 2a). Of these 11 serines, all but one are conserved in the mouse and rat Puma sequences, the serine at position 75 of the human sequence being replaced with a proline in both mouse and rat (Figure 2a). Cumulative serine-to-alanine mutations within Puma were produced by a series of site-directed mutagenesis reactions, in order to identify phosphorylation sites by a process of elimination. Several phosphorylation prediction programs (Netphos 2.0 (Technical University of Denmark), Disphos (Temple University, PA, USA) and Scansite (MIT, MA, USA)) were used to evaluate which serine residues had a higher chance of being phosphorylated, and these were grouped together for mutation to alanine (serines $9,10,36,96,106$ and 166), whereas the other serines $(33,75,83,98$ and 110) were mutated in a separate series of mutagenesis reactions. Mutation of serines $9,10,36,96,106$ and 166 to alanine completely prevented phosphorylation of Flag-Puma in HeLa cells, eliminating both the upper and lower phosphate-labelled bands (Figure 2b). In contrast, Puma in which serines 33, 75, 83, 98 and 110 had been mutated to alanines was phosphorylated in an identical manner to wild-type (WT) Puma. As expected, a construct in which all the serines within Puma were mutated to alanines was not phosphorylated (Figure 2b). These differences in phosphate labelling were not due to differences in expression, as all four Puma constructs used were efficiently immunoprecipitated when expressed in HeLa cells (Figure 2c). These results confirm the previous observation from the phosphoamino acid analysis that Puma is phosphorylated on serine residues, and show that serines $33,75,83,98$ and 110 are not targets for phosphorylation within HeLa cells. Subsequent experiments focused on revealing which of the serines $9,10,36,96,106$ and 166 were targets for phosphorylation. Further labelling reactions using different combinations of serine-to-alanine mutations allowed the sites of phosphorylation to be identified, which were subsequently confirmed using single-site mutants. Although the S9A (serine 9 to alanine) mutant was phosphorylated identically to WT-Puma, the S10A mutation completely ablated the lower phosphorylated band (Figure 2d) without affecting the upper band. Mutation of serines 96 and 106 specifically eliminated the upper phospho band (data not shown), without reducing the phosphorylation of the lower band. Although a S106A mutant also abrogated upper band phosphorylation, we could not definitively rule out a role for serine 96 in this phosphorylation event (Supplementary Figure 2), and this requires further investigation.

Phosphorylation of Puma at serines 10, 96 and 106 also occurs in MEF cells. To test whether Puma phosphorylation occurred in a different cell line and species, Bax/Bak double knockout mouse embryonic fibroblasts (DKO MEFs) were chosen for use in a series of labelling experiments similar to those performed in HeLa cells. DKO MEFs were chosen as deletion of Bax/Bak is known to prevent Pumainduced apoptosis, ${ }^{25,26}$ facilitating expression of exogenous Puma for IP. WT Puma was phosphorylated in DKO MEFs, with upper and lower phosphate-labelled bands mimicking 
a
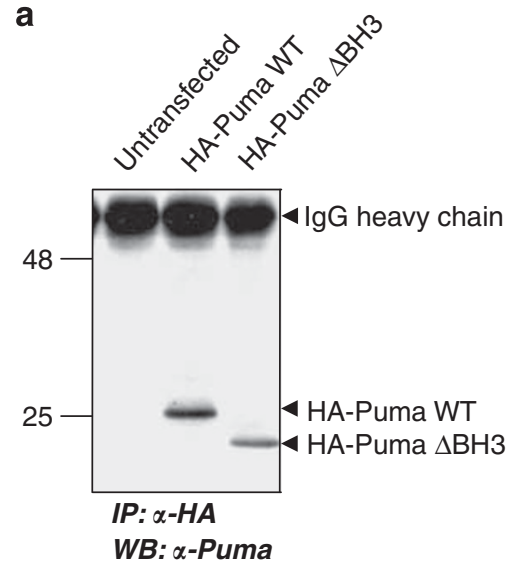

C

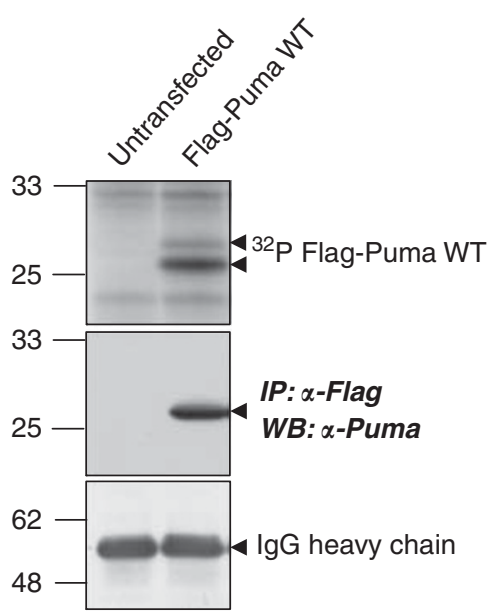

b
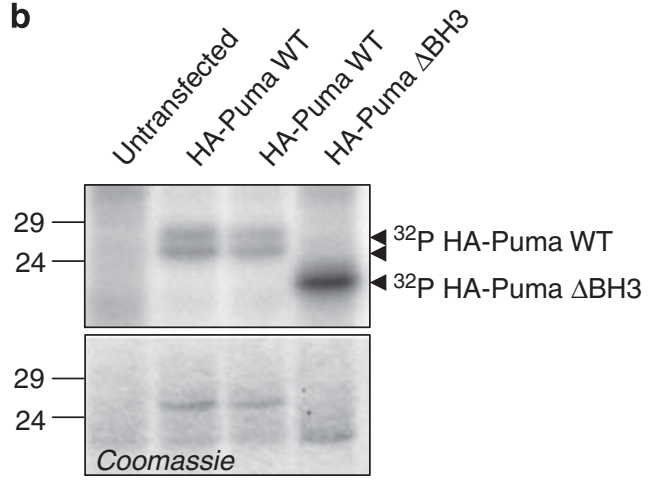

d

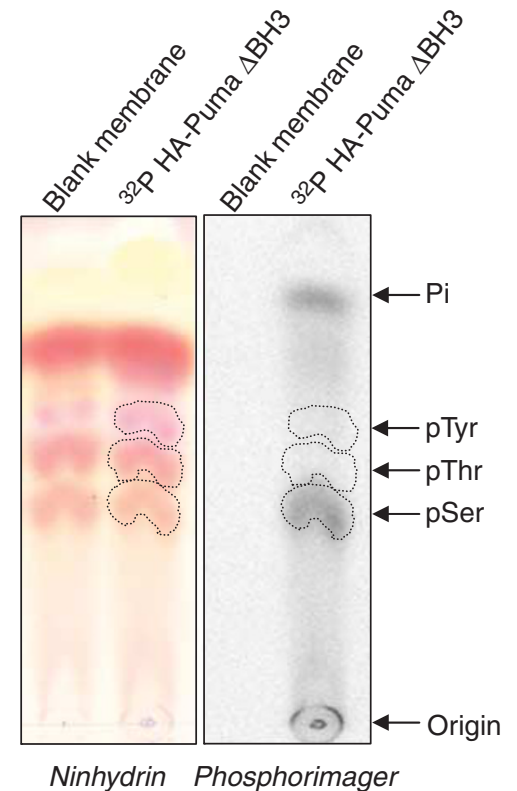

Figure 1 Exogenous Puma is phosphorylated on serine residues in HeLa cells in a BH3 domain-independent manner (a and $\mathbf{c}$ ) HeLa cells were transfected with a plasmid encoding HA-Puma (a) or Flag-Puma (c) and incubated in the presence of caspase inhibitor BAF for 16-20 h before lysis. Overexpressed Puma was immunoprecipitated using anti-HA antibody and protein A beads or anti-Flag beads. Immunoprecipitated Puma was detected using an anti-Puma N-terminus antibody. For analysis of Puma phosphorylation, cells were incubated for $4 \mathrm{~h}$ with ${ }^{32} \mathrm{P}$-orthophosphate before lysis. After SDS-PAGE, gels were stained with Coomassie to visualise protein and dried. ${ }^{32}$ P-labelled HA-Puma (b, upper panel) and Flag-Puma (c, upper panel) were detected using a phosphorimager system. HA-Puma-WT was run in duplicate in panel $\mathbf{b}$. (d) ${ }^{32}$ P-labelled HA-Puma was transferred to PVDF membrane. The radioactive Puma band was excised from the membrane and amino acids were obtained by acid hydrolysis. ${ }^{32} \mathrm{P}$-labelled amino acids were resuspended in $10 \mu \mathrm{l}$ milliQ water including phosphoamino acid standards and this sample was run on a 1D-TLC plate. Phosphoamino acid standards were visualised by ninhydrin staining, and ${ }^{32} \mathrm{P}$-labelled amino acids were detected using a phoshorimager system

the phosphorylation of Puma previously observed in HeLa cells (Figure 2e). Furthermore, mutation of S10A prevented ${ }^{32} \mathrm{P}$-labelling of the lower band and mutation of serine 96 and 106 to alanine eliminated the upper band. As with HeLa cells, mutation of all three sites was sufficient to completely prevent detectable phosphorylation of Puma in DKO MEFs (Figure 2e). This result indicates that the phosphorylation of Puma at serines 10, 96 and 106 is not a HeLa cell-specific phenomenon. These data therefore show that Puma is phosphorylated on several sites, the major site being serine 10 and the minor sites being serines 106 and/or 96, with these phosphorylations appearing to occur independently of one another. As the upper band was phosphorylated to a much lesser extent than the lower band, we focused our efforts on determining the functional effects of phosphorylation of the major site, that is, serine 10 .

Increased induction of apoptosis by S10A Puma compared with WT. To test whether mutation of serine 10 of Puma affected its apoptotic potency, expression constructs for WT, S10A and the non-apoptotic BH3 mutant, $\triangle \mathrm{LRR}$, were generated in the retroviral construct pBabe. This construct was chosen as its expression is driven from the relatively weak viral long-terminal repeat, thereby maximising the chances of identifying functional consequences of phosphorylation at Serine 10. pBabe-WT and pBabe-S10A were 


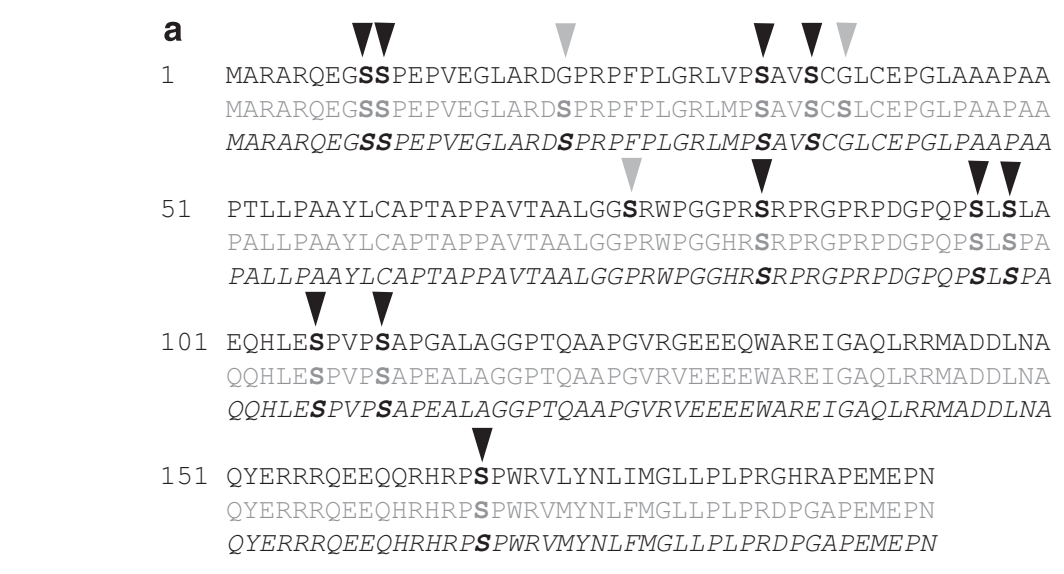

b
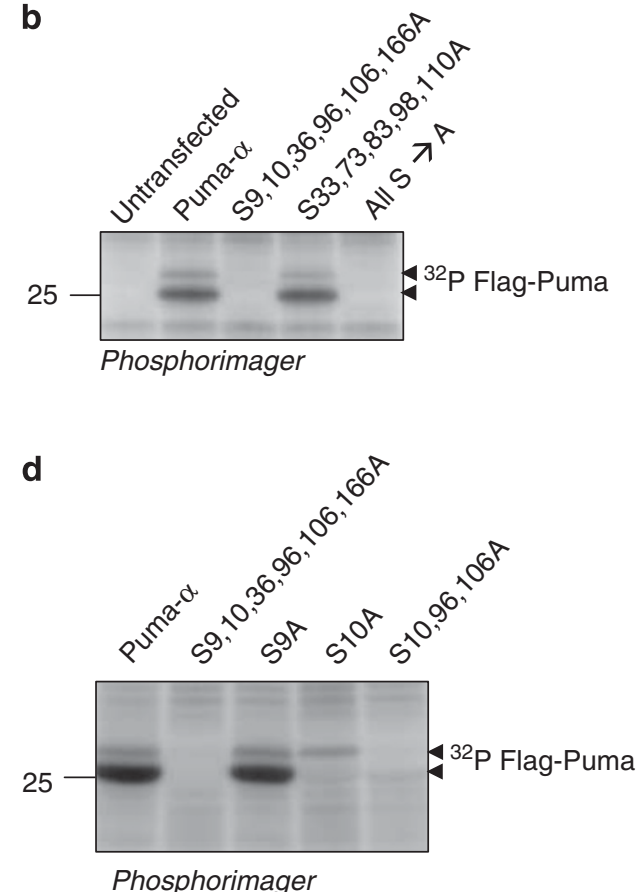

C

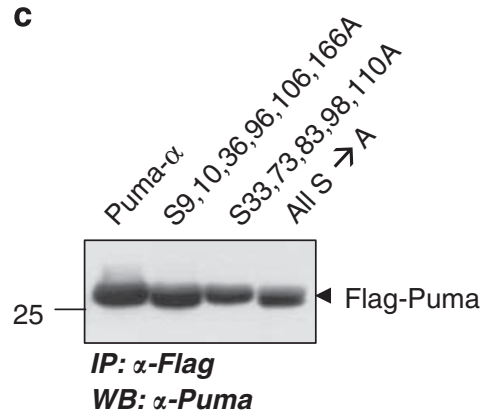

e

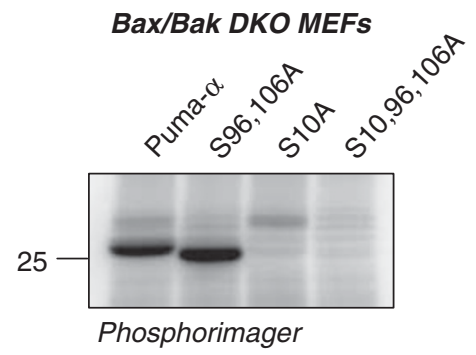

Figure 2 Serine 10 is the principle site of Puma phosphorylation in HeLa cells and MEFs. (a) Alignment of PUMA protein from human (black text), mouse (grey text) and rat (italics)-conserved serines are indicated by black arrowheads and non-conserved serines by grey arrowheads. HeLa cells (b and d) or Bax/Bak double knockout (DKO) MEFs (e) were transfected with a plasmid encoding Flag-Puma containing serine-to-alanine mutations at the sites indicated above each lane at $12 \mathrm{~h}$ before labelling with ${ }^{32} \mathrm{P}$-labelled orthophosphate. Flag-Puma was captured using anti-Flag beads, separated by SDS-PAGE and visualised by coomassie staining. ${ }^{32} \mathrm{P}$-labelled proteins were detected using a phosphorimager system. (c) Western blot detection of immunoprecipitated Puma mutants using an anti-Puma antibody

therefore co-transfected into HeLa cells with a vector encoding farnesylated GFP (fGFP). fGFP retains a strong fluorescent signal during later phases of apoptosis, which, along with evaluation of nuclear morphology by Hoechst 33342 staining, allowed quantification of dying cells amongst the transfected population. Of the GFP-positive population, $25 \%$ of those transfected with WT-Puma displayed apoptotic nuclei, in comparison with $11.8 \%$ in cells transfected with empty vector (Figure $3 a$ and b). As expected, transfection with the non-apoptotic BH3 mutant $\Delta$ LRR Puma did not induce any death above that seen with the empty vector (Figure $3 a$ and b). Importantly, mutation of serine 10 to alanine further enhanced the apoptosis associated with Puma expression, with a further $9.2 \%$ of the transfected population undergoing death compared with WT at the $20 \mathrm{~h}$ time point (Figure $3 a$ and $b, S 10 A=35.0 \%$ death).

Non-phosphorylatable serine $\mathbf{1 0}$ mutants are stabilised compared with WT serine 10. As phosphorylation of the $\mathrm{BH} 3$ proteins Bim and Bad is known to modulate binding to anti-apoptotic binding partners, ${ }^{20-22}$ we tested whether mutation of serine 10 altered the ability of Puma to bind Bcl-2, $\mathrm{BCl}-\mathrm{xL}$ and $\mathrm{Mcl}-1$, thereby explaining the difference in apoptotic potential between this mutant and the WT protein. Puma-S10A was, however, still able to bind these anti-apoptotic members of the Bcl-2 family in keeping with the position of serine 10 being far from the $\mathrm{BH} 3$ domain (Figure 4). 

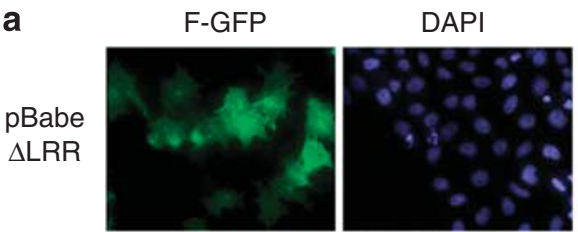

overlay
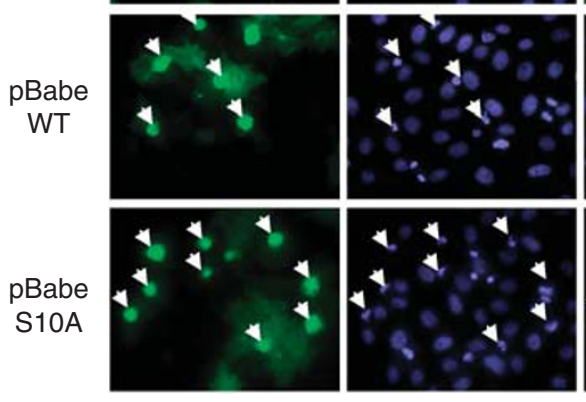

b

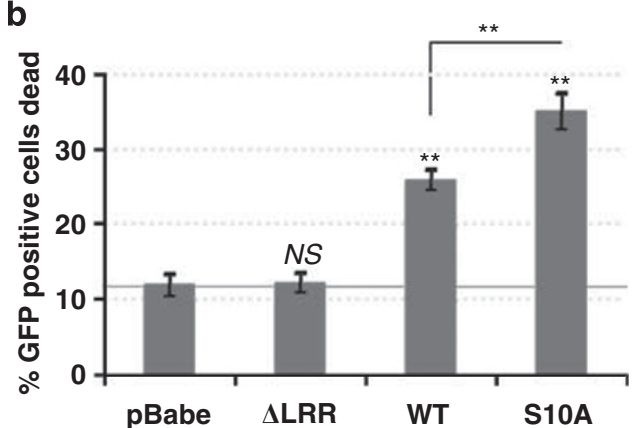

Figure 3 Non-phosphorylatable Puma mutant S10A induces apoptosis at a greater rate than WT-Puma in HeLa cells. ( $\mathbf{a}$ and $\mathbf{b}$ ) HeLa cells were co-transfected with the indicated Puma construct and a construct encoding farnesylated GFP. At $16 \mathrm{~h}$ after transfection, cells were fixed with $4 \%$ PFA for 20 min, DNA was stained with Hoechst 33342 and then coverslips mounted for analysis by fluorescence microscopy (a). Cell death was quantified by examining the nuclear morphology of GFP-positive cells and calculating the percentage displaying condensed or fragmented apoptotic nuclei (white arrowheads) (b). ${ }^{*} P<0.01$

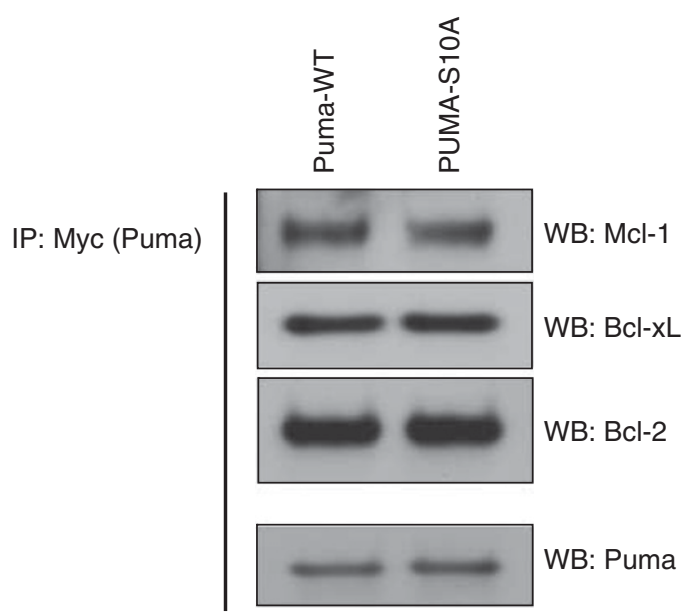

Figure 4 Mutation of Puma serine 10 does not affect association of Puma with Mcl-1, Bcl-2 or Bcl-xL. Cells expressing Myc-tagged Puma-WT or Puma-S10A were immunoprecipitated with anti-Myc antibody and precipitated proteins were analysed by western blotting with antibodies against: $\mathrm{Mcl}-1, \mathrm{Bcl}-2, \mathrm{Bcl}-\mathrm{Xl}$ and Puma
It has also been reported that mitochondrial localisation has an important role in the induction of death by Puma, although this is not always the case. ${ }^{26,27}$ Phosphorylation can also alter the subcellular localisation of the BH3-only proteins Bim and Bad, thus affecting their apoptotic potency. ${ }^{20,24,28,29}$ To examine whether mutation of serine 10 altered the subcellular localisation of Puma, we performed immunofluorescence on HeLa cells and Bax/Bak DKO MEFs expressing Puma-WT or Puma-S10A. In both HeLa and DKO MEF cells, WT and S10A Puma co-localised to an equal extent with the mitochondrial markers, mitotracker and TOM20 (Supplementary Figure 3).

We next analysed the expression levels of WT and S10A by western blotting. This revealed that S10A was expressed at a higher steady state level than WT Puma (Figure 5a). This difference was not due to transfection efficiency or different transcription rates from the two plasmids, as equal amounts of Puma mRNA could be detected by qPCR in the WT and mutant Puma-expressing cells (Figure 5b).

Relatively little has been published regarding the regulation of Puma protein stability. A recent paper from Callus et al. ${ }^{30}$ showed, however, that after induction of MOMP and caspase activation by expression of Puma from an inducible promoter, Puma was degraded in a caspase-dependent manner. Consequently, the authors showed that co-expression of $\mathrm{Bcl}-2$ to prevent MOMP or expression of Puma in Bax/Bak-null cells that do not undergo MOMP (Wei et al. ${ }^{10}$ and Supplementary Figure 3 ) resulted in increased levels of Puma protein expression. ${ }^{30}$ We therefore tested whether these factors could affect the expression of WT Puma and the Puma-S10A in our cell systems. When Puma was expressed in WT MEFs, the presence of the pan-caspase inhibitor zVAD-fmk did not result in any change in WT-Puma or S10A protein levels, indicating that the difference in expression of Puma-WT and Puma-S10A is not dependent on caspase activity (Figure $5 \mathrm{c}$, left panels). We also generated serine 10 mutants in which three conserved amino acids within the $\mathrm{BH} 3$ domain of Puma were mutated $(\Delta L R R)$, thus eliminating binding to anti-apoptotic $\mathrm{Bcl}-2$ proteins and rendering the protein non-apoptotic. S10A- $\Delta$ LRR still showed higher steady state levels than WT $\Delta \mathrm{LRR}$, confirming that the increased expression of S10A compared with WT protein was independent of anti-apoptotic Bcl-2 protein binding, MOMP induction and subsequent caspase activation (Figure $5 \mathrm{~d}$ ).

Puma has also recently been shown to induce degradation of mitochondria by autophagy in a BH3-dependent, Bax/Bakdependent manner. ${ }^{31}$ Given that Puma itself is a mitochondrially targeted protein, this raises the possibility that Puma may trigger its own turnover by autophagic processes. However, expression of Puma in MEFs lacking classical macroautophagy due to genetic deletion of Atg5 did not affect the relative expression of S10A compared with WT, when compared with WT MEFs either in the absence or presence of the pan-caspase inhibitor zVAD-fmk. These findings therefore rule out a role for macroautophagy in Puma turnover and the selective modulation of Puma serine 10 mutant stability in our cell systems (Figure 5c).

Turnover of the non-phosphorylatable S10A Puma is delayed compared with the WT protein. As Puma was not turned over by caspases or by autophagy in our cell systems, 


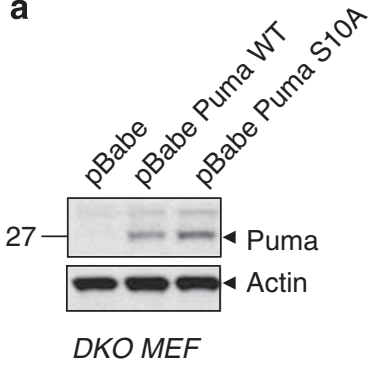

b

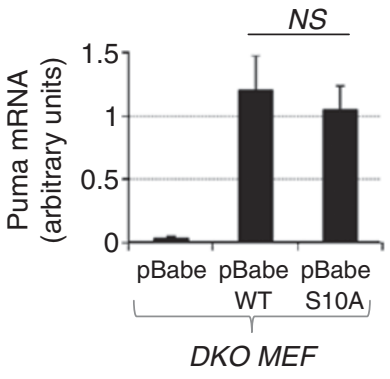

C

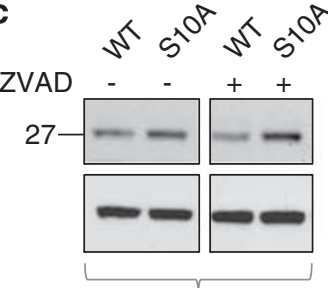

WT MEF

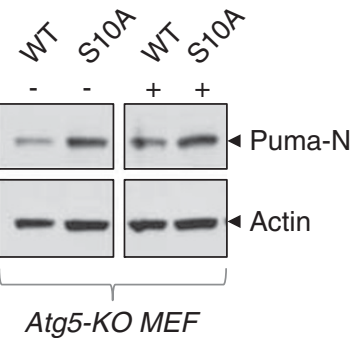

d

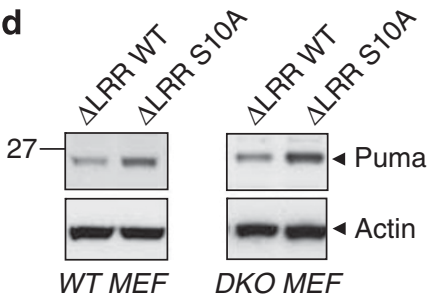

Figure 5 The non-phosphorylatable Puma serine 10 mutant accumulates in a manner independent of caspases, apoptosis and autophagy. (a) DKO MEFs were lysed $24 \mathrm{~h}$ after transfection with the indicated constructs. Proteins were resolved by SDS-PAGE and Puma protein was detected using an antibody directed against human Puma. (b) qRT-PCR analysis of DKO MEFs transfected for $20 \mathrm{~h}$ with the indicated constructs. The amount of Puma mRNA was normalised in each sample against $18 \mathrm{~S}$ RNA. (c) WT or ATG-5 KO MEFs were transfected with either WT or S10A Puma constructs in the presence or absence of pan-caspase inhibitor zVAD-fmk. At 24h after transfection, cells were lysed and Puma expression analysed by western blot using a Puma N-terminal antibody. (d) WT and DKO MEFs were transfected with non-apoptotic Puma lacking a functional $\mathrm{BH} 3$ motif $(\Delta \mathrm{LRR})$ containing either the wild-type serine at position 10 or an alanine substitution. At $24 \mathrm{~h}$ after transfection, cells were lysed and Puma expression was analysed by western blot analysis using anti-Puma antibodies

Time after addition of cycloheximide (hours)

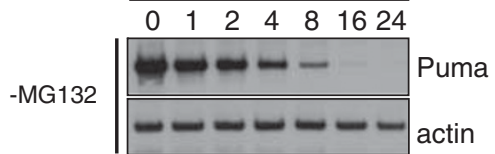

+MG132

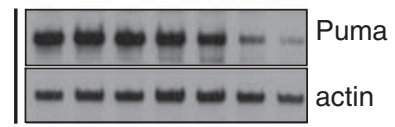

b

Un WT

S10A

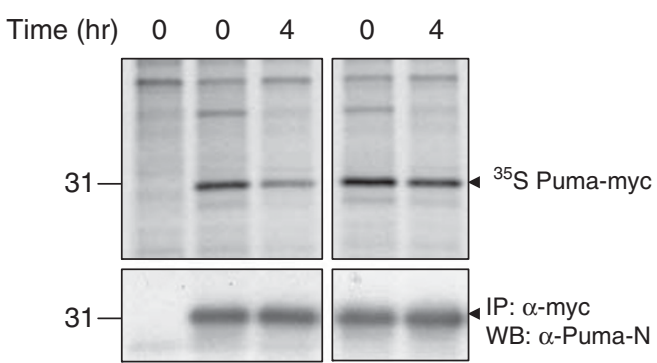

C

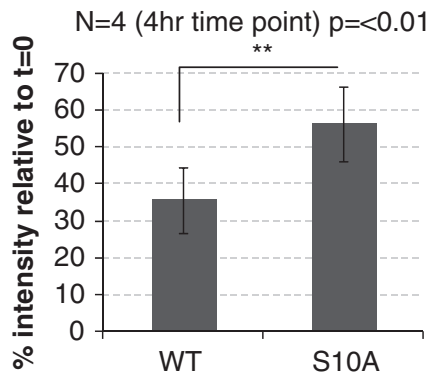

Figure 6 WT-Puma is degraded faster than non-phosphorylatable Puma S10A. (a) Puma is degraded by the proteasome. DKO MEFs infected with Myc-tagged wild-type Puma were treated with cycloheximide either in the absence or presence of the proteasome inhibitor MG132. At the indicated time periods, cell lysates were prepared and analysed by western blotting with antibodies against Puma and actin. (b) DKO MEFs were transfected with Myc-tagged Puma constructs as indicated. Cells were pulsed for $1 \mathrm{~h}$ with $50 \mu \mathrm{Ci}{ }^{35} \mathrm{~S}$-labelled methionine/cysteine before a $4 \mathrm{~h}$ chase step in medium containing excess cold methionine/cysteine. Cells were lysed at the indicated time points after the pulse and Myc-tagged Puma immunoprecipitated. Puma expression was tested by western blot (b, lower panel) and the amount of ${ }^{35} \mathrm{~S}$ label incorporated into Puma determined using a phosphorimager system. (c) Intensities of ${ }^{35} \mathrm{~S}$-labelled-Puma from four separate experiments were quantified by measuring band intensity using ImageJ ( $\mathrm{NIH}$, Bethesda, MD, USA) and are expressed as a percentage of the band intensity of WT or S10A at $t=0 .{ }^{* *} P<0.01$

we next tested whether Puma was targeted for degradation at the proteasome. Cells expressing WT Puma were therefore treated with cycloheximide, and the rate of Puma decay monitored either in the absence or presence of the proteasome inhibitor, MG132. This assay revealed that degradation of Puma was retarded in the presence of MG132, indicating a role for the proteasome in Puma turnover and stability (Figure 6a).

As these results indicate that Puma is actively turned over in a proteasomal-dependent manner, we reasoned that the increased amount of S10A protein observed may be caused by differences in the rate of turnover caused by phosphorylation of the WT protein. To test this possibility, we performed ${ }^{35} \mathrm{~S}$ pulse-chase analysis to measure the rate of degradation of WT Puma and S10A in Bax/Bak DKO MEFs. Following a $1-h^{35} S$ pulse and a 4-h chase, a clear decrease in the amount of ${ }^{35}$ S-labelled Puma-WT protein was observed, consistent with it being degraded (Figure 6b). Although there was a small decrease in ${ }^{35}$ S-labelled Puma-S10A during this period, this decrease was markedly lower than the decrease observed with the WT protein, with $56 \%$ of the S10A remaining relative to $t=0$ compared with $35 \%$ of the WT (Figure $6 \mathrm{c}$ ). Thus, the phosphorylation site at serine 10 has an important role in determining the rate of turnover of Puma, with the 
non-phosphorylatable S10A mutant being degraded at a slower rate than the WT protein.

\section{Discussion}

To our knowledge, this is the first report to show that the $\mathrm{BH}$ only protein Puma is subject to post-translational modification by phosphorylation. We show that multiple serines are phosphorylated on Puma with the major site being serine 10. To date, previous studies have principally shown that Puma is transcriptionally upregulated by pro-apoptotic factors such as p53, p73, E2F and FOXO3a. ${ }^{12,13,17-19}$ However, as not all cells undergo a cell death response after up-regulation of Puma by these transcription factors, this suggests that other mechanisms may be at play to suppress the potent death-inducing activity of Puma in cells. In this regard, it is of interest that the potency of other BH3-only proteins, for example, Bim and Bad, is reduced by phosphorylation induced by survival signalling pathways. ${ }^{20,22}$ This also seems to be the case for Puma, as inhibition of phosphorylation at serine 10 of Puma increases the pro-apoptotic potential of the protein. Previous reports relating to phosphorylation of other $\mathrm{BH} 3-$ only proteins have shown that this can affect interaction of the BH3-only protein with anti-apoptotic members of the Bcl2 family. In the case of Puma, however, this seems not to be the case, as equivalent levels of interaction were found between anti-apoptotic members of the Bcl2 and either WT Puma or a mutant of Puma in which serine 10 had been mutated to alanine. In contrast, it was found that mutation of serine 10 increased the half-life of the Puma, indicating that phosphorylation at this serine destabilises the protein, thereby keeping its death-inducing potential in check.

Although what we report here represents a definitive body of work highlighting a new regulatory step in Puma function, a number of questions are raised that are worthy of discussion. First, it is important to consider which cellular signalling pathways impinge on serine 10 to regulate Puma function. In the case of Bad and Bim, classic survival pathways are involved directly in their phosphorylation, such as Akt and mitogen-activated protein kinase (MAPK) pathways, respectively. ${ }^{20,22}$ As serine 10 of Puma is immediately followed by a proline, which indicates a potential MAPK phosphorylation site, we considered that Puma may also be affected by MAPK kinase signalling. However, treatment of cells with inhibitors of p38, JNK and mitogen-activated protein kinase kinase (the kinase that modulates the activity of the Erk1 and Erk2 MAPKs) had no effect on Puma stability or phosphorylation (data not shown). In light of this, cells were treated with a large broader spectrum panel of kinase inhibitors, but no effects on Puma stability were observed (data not shown). Therefore, despite extensive efforts to identify a cellular signalling pathway that affects Puma stability through serine 10 phosphorylation, the kinase(s) responsible for this effect remain to be defined. In addition, although beyond the scope of the study presented here, it is also important to consider how the other phosphorylation sites we have identified in Puma, and the signalling pathways with which they connect, may also affect Puma function. For instance, do these sites work in concert with serine 10 to modulate Puma stability or are they involved, as outlined above, in regulating $\mathrm{BH} 3-$ only function by controlling binding to anti-apoptotic $\mathrm{Bcl} 2$ family proteins. Further studies in this area are undoubtedly worthwhile and may also reveal further novel insights into the regulation of Puma function.

In summary, the studies presented here highlight a novel and potentially extremely important mechanism by which cells can restrain one of the most potent death-inducing proteins they contain. Through further investigation, our findings also potentially open up new possibilities, whereby we can modulate the death-inducing potential of Puma, either positively or negatively, for therapeutic gain.

\section{Materials and Methods}

Cell culture and constructs. Cell lines were cultured in Dulbecco's modified Eagle's medium (DMEM) (Invitrogen, Paisley, UK) supplemented with $100 \mathrm{U} / \mathrm{ml}$ penicillin G, $100 \mu \mathrm{g} / \mathrm{ml}$ streptomycin sulphate (Invitrogen) and 10\% fetal bovine serum (FBS) (Sigma, St Louis, MO, USA). Cells were maintained at $37^{\circ} \mathrm{C}$ in $5 \%$ $\mathrm{CO}_{2}$-balanced air. HeLa cells were obtained from ATCC (Manassas, VA, USA). Bax/Bak DKO MEFs and Atg-5 knockout MEFs were kind gifts from Stanley Korsmeyer (Dana-Farber Institute, Boston, MA, USA) and Noboru Mizushima (Tokyo Medical and Dental University, Tokyo, Japan), respectively. DKO cell lines stably expressing Puma and Puma mutants were generated as previously described by retroviral infection of the pBabe-puro constructs outlined below. ${ }^{32}$ HA-tagged Puma- $\alpha$ and flag-tagged Puma- $\alpha$ constructs were generously provided by Bert Vogelstein (HHMI, Johns Hopkins, Baltimore, MD, USA) and Karen Vousden (Beatson Institute for Cancer Research, Glasgow, UK). To generate untagged, $\mathrm{N}$-terminally flag-tagged or C-terminally Myc-tagged Puma constructs, Puma was inserted into vectors pcDNA3.1, pcDNA3.1Myc/His and pBabe-puro by PCR amplification of flag-tagged or untagged Puma sequences. All vector insertions were verified by DNA sequencing.

Immunoprecipitation. HeLa cells or DKO MEFs were transfected using Lipofectamine 2000 (Invitrogen) or Fugene 6 (Roche, Basel, Switzerland) following the manufacturer's instructions, and proteins were allowed to express for 16-20 h. HeLa cells were treated with pan-caspase inhibitor BAF $(50 \mu \mathrm{M})$ during this period to prevent apoptotic death caused by Puma expression. Cells were lysed in $500 \mu \mathrm{l}$ lysis buffer (50 mM Tris-HCl (pH 7.5), $1 \% \mathrm{NP}-40,120 \mathrm{mM} \mathrm{NaCl}, 1 \mathrm{mM}$ EDTA, $25 \mathrm{mM} \mathrm{NaF}, 40 \mathrm{mM} \beta$-glycerolphosphate, $1 \mathrm{mM}$ sodium orthovanadate, $1 \times$ complete protease inhibitor cocktail (Roche)) and insoluble debris removed by centrifugation at 10000 r.p.m. at $4^{\circ} \mathrm{C}$ for $10 \mathrm{~min}$. For HA-tag IP, $2 \mu \mathrm{g}$ of anti-HA antibody was added (sc-805, Santa Cruz Biotechnology, Santa Cruz, CA, USA) and incubated with the sample for $1 \mathrm{~h}$ at $4^{\circ} \mathrm{C}$ followed by a further addition of $50 \mu \mathrm{l}$ of a $50 \%$ slurry of protein A sepharose beads (Sigma) and incubation overnight at $4{ }^{\circ} \mathrm{C}$. For Flag-tag IP, $30 \mu \mathrm{l}$ of anti-Flag M2 affinity gel (Sigma) was added and lysates incubated overnight at $4^{\circ} \mathrm{C}$ as previously described.$^{33}$ For Myc-tag IP, $4 \mu \mathrm{g}$ of $4 \mathrm{~A} 6$ monoclonal Myc antibody was added (Millipore, Billerica, MA, USA) and incubated with the sample for $1 \mathrm{~h}$ at $4^{\circ} \mathrm{C}$ followed by a further addition of $50 \mu \mathrm{l}$ of a $50 \%$ slurry of protein $\mathrm{G}$ sepharose beads (Sigma). Beads were washed four times in ice-cold lysis buffer before being resuspended in $30 \mu \mathrm{l}$ of $1 \times$ gel-loading buffer $(50 \mathrm{mM}$ Tris- $\mathrm{HCl}$ (pH 6.8), $100 \mathrm{mM} \mathrm{DTT,} 2 \%$ SDS, $10 \%$ glycerol and bromophenol blue). Captured proteins were eluted by boiling for $5 \mathrm{~min}$ and eluates loaded onto appropriate SDS-PAGE gels.

Western blot analysis. For western blot analysis, $40 \mu \mathrm{g}$ protein lysate per lane or protein eluate from IP were separated on 10-15\% SDS-polyacrylamide gels as previously described. ${ }^{34}$ Proteins were transferred to $0.22 \mu \mathrm{M}$ pore nitrocellulose membranes (GE Healthcare, Littlechalfort, UK). Primary antibodies were diluted in TBS-T as follows: Puma anti-N-terminus (Prosci Inc, Poway, CA, USA; 1: 1000), Puma anti-C-terminus (Sigma; $1: 500$ ) and $\beta$-actin (Abcam, Cambridge, MA, USA; $1: 5000)$. Signal was detected using an appropriate HRP-conjugated secondary antibody and ECL reagents (GE Healthcare).

Metabolic labelling of cells with inorganic ${ }^{32} \mathbf{P}$. At $12-16 \mathrm{~h}$ after transfection, culture medium was replaced with phosphate-free DMEM (MP Biomedicals, Solon, $\mathrm{OH}$, USA) supplemented with $10 \% \mathrm{FBS}$ and $0.5 \mathrm{mCi}{ }^{32} \mathrm{P}$-labelled orthophosphate (GE Healthcare). Cells were labelled for $4 \mathrm{~h}$ in a humidified atmosphere of $5 \% \mathrm{CO}_{2}$. After labelling, cells were lysed and IPs performed as 
described above, before proteins were separated by SDS-PAGE. Gels were stained for total protein in 50\% methanol, $10 \%$ acetic acid (Sigma) and $0.1 \%$ Coomassie R-250 (Sigma) and destained in 50\% methanol. Once destained, gels were dried using an ATTO RapiDry system (ATTO, Tokyo, Japan), and ${ }^{32} \mathrm{P}$-labelled proteins were then detected using a phosphorimager system (Molecular Dynamics, Sevenoaks, UK).

Phosphoamino acid analysis. For the purposes of phosphoamino acid analysis, ${ }^{32}$ P-labelled proteins were separated by SDS-PAGE as described above and electroblotted onto a PVDF (Immobilon P, Millipore) membrane. Total protein was visualised by staining with $0.1 \%$ India Ink in PBS and $0.3 \%$ Tween. Radiolabelled proteins were detected using the phosphorimager as before, and hot protein bands were excised from the PVDF membrane using a sterile scalpel. After acid hydrolysis in $6 \mathrm{M} \mathrm{HCl}$ at $110^{\circ} \mathrm{C}$ for $1 \mathrm{~h}$, the sample was cooled and the liquid hydrolysate was transferred to a fresh tube and dried using a SpeedVac (Thermo Fisher Scientific, Waltham, MA, USA). Sample was redissolved in $10 \mu \mathrm{l} \mathrm{milliQ} \mathrm{H}_{2} \mathrm{O}$ and spotted onto a plastic-backed cellulose TLC plate. Phosphoserine, -threonine or -tyrosine standards $(10 \mu \mathrm{g})$ were run alongside samples using butanol/acetic acid/ ethanol/ $/ \mathrm{H}_{2} \mathrm{O}(1: 1: 1: 1)$ as the mobile phase. Phosphoamino acid standards were visualised by staining with $0.25 \%(\mathrm{w} / \mathrm{v})$ ninhydrin in acetone and radiolabelled phosphoamino acids were detected using the phosphorimager system.

Site-directed mutagenesis. The 13 serine residues in human Puma- $\alpha$ were replaced with alanine residues using the Quikchange multi-site-directed mutagenesis kit (Agilent Technologies, Santa Clara, CA, USA) according to the manufacturer's instructions. Mutagenic primers were designed to anneal to the same strand of template DNA, have a minimum GC content of $40 \%$, terminate with at least one $\mathrm{G}$ or $\mathrm{C}$, be between 25 and 45 base pairs long and have a melting temperature of over $75^{\circ} \mathrm{C}$ as calculated using the tools available in the website http://www.stratagene.com.

qPCR analysis. Total RNA was extracted from cells using the Qiagen RNeasy Mini Kit (Qiagen, Hilden, Germany) following the manufacturer's instructions. RNA was eluted in RNase-free water and stored at $-80^{\circ} \mathrm{C}$. Total RNA was quantified by absorbance at $260 \mathrm{~nm}$ (Nanodrop Technologies, Wilmington, DE, USA), and $0.5 \mu \mathrm{g}$ of RNA from each sample was used for cDNA synthesis using the DyNAmo kit (Thermo Fisher Scientific). Gene-specific oligonucleotides were obtained from Qiagen, and SYBR Green was used to detect Taq-amplified products. qPCR reactions were carried out as previously described.$^{35} \mathrm{All}$ signals were normalised against $18 \mathrm{~S}$ rRNA.

Quantification of cell death. HeLa cells plated on glass coverslips were transfected with a 1:1 ratio of Puma and fGFP expression plasmids. At $16 \mathrm{~h}$ posttransfection, cells were fixed in 3\% PFA and nuclei-stained using Hoechst 33342 before mounting on slides. GFP-positive cells were counted and cell death in the GFPpositive population evaluated by nuclear and cellular morphology. As expected, cell death was not induced by a mutant form of Puma lacking a functional $\mathrm{BH} 3$ domain and was inhibited by the pan-caspase inhibitor ZVAD-fmk (not shown).

Immunocytochemistry. Cells were fixed in $3 \%$ paraformaldehyde for $20 \mathrm{~min}$ at room temperature and coverslips were then washed three times in ice-cold PBS. Cells were permeabilised for $30 \mathrm{~min}$ at room temperature in PBS and $0.3 \%$ Triton X-100 (PBSTX) (VWR, West Chester, PA, USA). Coverslips were incubated with primary antibodies overnight at $4{ }^{\circ} \mathrm{C}$. Primary antibodies were diluted as follows: Puma anti-N-terminus (Prosci Inc; 1 : 500), Tom 20 (BD Biosciences, San Hose, CA, USA, no. 612278; $1: 1000)$. After four washes in PBSTX, coverslips were incubated with appropriate fluorescence-conjugated secondary antibodies at room temperature for $1 \mathrm{~h}$. Cells were washed a further two times with PBS before a brief incubation with $5 \mu \mathrm{g} / \mathrm{ml}$ Hoechst 33342 (Sigma) and further washes in PBS. Coverslips were mounted using Fluoromount-G (Southern Biotech, Birmingham, AL, USA) and analysed by confocal microscopy (IX70 connected to UltaVIEW, LCl confocal imaging system, Perkin Elmer, Waltham, MA, USA).

Metabolic labelling of cells with ${ }^{35} \mathrm{~S}$ methionine/cysteine for pulse-chase analysis. At $16 \mathrm{~h}$ after transfection with myc-tagged Puma constructs, DKO MEFs were placed in DMEM lacking methionine and cysteine supplemented with $10 \%$ dialysed FBS for $1 \mathrm{~h}$ before addition of $50 \mu \mathrm{Ci}$ of ${ }^{35} \mathrm{~S}$-labelled methionine/cysteine (PerkinElmer). After a $60 \mathrm{~min}$ pulse, cells were washed 3 times in PBS and then chased in DMEM containing excess cold methionine and cysteine $(2 \mathrm{mM})$ for the time periods indicated. Cells were lysed and myc-tagged Puma was captured by IP as described above. Immunoprecipitated proteins were separated by SDS-PAGE, transferred to nitrocellulose membranes for detection of Puma by western blot followed by exposure to a phosphorimager screen in order to detect the amount of ${ }^{35} \mathrm{~S}$ signal.

\section{Conflict of interest}

The authors declare no conflict of interest.

Acknowledgements. We thank Noboru Mizushima, Craig Thompson, Andy Villunger, Stanley Korsmeyer, Bert Vogelstein and Karen Vousden for reagents and members of the Tumour Cell Death Laboratory for critical reading of the paper. Work in the Tumour Cell Death Laboratory is supported by Cancer Research UK and the Association for International Cancer Research. Work in the Tolkovsky Laboratory was supported by the Wellcome Trust. MF was the recipient of a BBSRC research studentship (University of Cambridge) and was supported by Cancer Research UK as a post-doctoral fellow (Beatson Institute).

1. van Delft MF, Huang DC. How the Bcl-2 family of proteins interact to regulate apoptosis. Cell Res 2006; 16: 203-213.

2. Cory S, Adams JM. The Bcl2 family: regulators of the cellular life-or-death switch. Nat Rev Cancer 2002; 2: 647-656.

3. Giam M, Huang DC, Bouillet P. BH3-only proteins and their roles in programmed cell death. Oncogene 2008; 27 (Suppl 1): S128-S136.

4. Green DR, Kroemer G. The pathophysiology of mitochondrial cell death. Science 2004; 305: 626-629.

5. Danial NN, Korsmeyer SJ. Cell death: critical control points. Cell 2004; 116: 205-219.

6. Willis SN, Adams JM. Life in the balance: how BH3-only proteins induce apoptosis. Curr Opin Cell Biol 2005; 17: 617-625.

7. Letai A, Bassik MC, Walensky LD, Sorcinelli MD, Weiler S, Korsmeyer SJ. Distinct BH3 domains either sensitize or activate mitochondrial apoptosis, serving as prototype cancer therapeutics. Cancer Cell 2002; 2: 183-192.

8. Cartron PF, Gallenne T, Bougras G, Gautier F, Manero F, Vusio P et al. The first alpha helix of Bax plays a necessary role in its ligand-induced activation by the $\mathrm{BH}$-only proteins Bid and PUMA. Mol Cell 2004; 16: 807-818.

9. Kim H, Rafiuddin-Shah M, Tu HC, Jeffers JR, Zambetti GP, Hsieh JJ et al. Hierarchical regulation of mitochondrion-dependent apoptosis by BCL-2 subfamilies. Nat Cell Biol 2006; 8: 1348-1358.

10. Wei MC, Zong WX, Cheng EH, Lindsten T, Panoutsakopoulou V, Ross AJ et al. Proapoptotic BAX and BAK: a requisite gateway to mitochondrial dysfunction and death. Science 2001; 292: 727-730.

11. Lomonosova $\mathrm{E}$, Chinnadurai $\mathrm{G}$. BH3-only proteins in apoptosis and beyond: an overview. Oncogene 2008; 27 (Suppl 1): S2-S19.

12. Nakano K, Vousden KH. PUMA a novel proapoptotic gene, is induced by p53. Mol Cell 2001; 7: 683-694

13. Yu J, Zhang L, Hwang PM, Kinzler KW, Vogelstein B. PUMA induces the rapid apoptosis of colorectal cancer cells. Mol Cell 2001; 7: 673-682.

14. Han J, Flemington C, Houghton AB, Gu Z, Zambetti GP, Lutz RJ et al. Expression of bbc3, a pro-apoptotic $\mathrm{BH} 3-$ only gene, is regulated by diverse cell death and survival signals. Proc Natl Acad Sci USA 2001; 98: 11318-11323.

15. Yu J, Zhang L. PUMA a potent killer with or without p53. Oncogene 2008; 27 (Suppl 1): S71-S83.

16. Villunger A, Michalak EM, Coultas L, Mullauer F, Bock G, Ausserlechner MJ et al. p53- and drug-induced apoptotic responses mediated by $\mathrm{BH} 3-$ only proteins Puma and noxa. Science 2003; 302: 1036-1038.

17. Melino G, Bernassola F, Ranalli M, Yee K, Zong WX, Corazzari $M$ et al. p73 Induces apoptosis via PUMA transactivation and Bax mitochondrial translocation. J Biol Chem 2004; 279: 8076-8083.

18. You H, Pellegrini M, Tsuchihara K, Yamamoto K, Hacker G, Erlacher M et al. FOXO3adependent regulation of Puma in response to cytokine/growth factor withdrawal. J Exp Med 2006; 203: 1657-1663.

19. Hershko T, Ginsberg D. Up-regulation of $\mathrm{Bcl}-2$ homology $3(\mathrm{BH} 3)$-only proteins by E2F1 mediates apoptosis. J Biol Chem 2004; 279: 8627-8634.

20. Zha J, Harada H, Yang E, Jockel J, Korsmeyer SJ. Serine phosphorylation of death agonist BAD in response to survival factor results in binding to 14-3-3 not BCL-X(L). Cell 1996; 87: 619-628.

21. Zhou XM, Liu Y, Payne G, Lutz RJ, Chittenden T. Growth factors inactivate the cell death promoter BAD by phosphorylation of its BH3 domain on Ser155. J Biol Chem 2000; 275: 25046-25051.

22. Ewings KE, Hadfield-Moorhouse K, Wiggins CM, Wickenden JA, Balmanno K, Gilley $R$ et al. ERK1/2-dependent phosphorylation of BimEL promotes its rapid dissociation from Mcl-1 and Bcl-xL. EMBO J 2007; 26: 2856-2867.

23. Verma S, Zhao LJ, Chinnadurai G. Phosphorylation of the pro-apoptotic protein BIK: mapping of phosphorylation sites and effect on apoptosis. J Biol Chem 2001; 276 4671-4676. 
24. Lei K, Davis RJ. JNK phosphorylation of Bim-related members of the Bcl2 family induces Bax-dependent apoptosis. Proc Natl Acad Sci USA 2003; 100: 2432-2437.

25. Ming L, Wang P, Bank A, Yu J, Zhang L. PUMA dissociates Bax and $B c l-X(L)$ to induce apoptosis in colon cancer cells. J Biol Chem 2006; 281: 16034-16042.

26. Yu J, Wang Z, Kinzler KW, Vogelstein B, Zhang L. PUMA mediates the apoptotic response to p53 in colorectal cancer cells. Proc Natl Acad Sci USA 2003; 100: 1931-1936.

27. Yee KS, Vousden KH. Contribution of membrane localization to the apoptotic activity of PUMA. Apoptosis 2008; 13: 87-95.

28. Qi XJ, Wildey GM, Howe PH. Evidence that Ser87 of BimEL is phosphorylated by Akt and regulates BimEL apoptotic function. J Biol Chem 2006; 281: 813-823.

29. Puthalakath $\mathrm{H}$, Huang DC, O'Reilly LA, King SM, Strasser A. The proapoptotic activity of the Bcl-2 family member Bim is regulated by interaction with the dynein motor complex. Mol Cell 1999; 3: 287-296.

30. Callus BA, Moujallad DM, Silke J, Gerl R, Jabbour AM, Ekert PG et al. Triggering of apoptosis by Puma is determined by the threshold set by prosurvival Bcl-2 family proteins. J Mol Biol 2008; 384: 313-323.

31. Yee KS, Wilkinson S, James J, Ryan KM, Vousden KH. PUMA- and Bax-induced autophagy contributes to apoptosis. Cell Death Differ 2009; 16: 1135-1145.
32. O'Prey J, Crighton D, Martin AG, Vousden KH, Fearnhead HO, Ryan KM. p53-mediated induction of Noxa and p53AIP1 requires NFkappaB. Cell Cycle 2010; 9: 947-952.

33. Bell HS, Dufes C, O'Prey J, Crighton D, Bergamaschi D, Lu X et al. A p53-derived apoptotic peptide derepresses p73 to cause tumor regression in vivo. J Clin Invest 2007; 117: 1008-1018.

34. Bell LA, O'Prey J, Ryan KM. DNA-binding independent cell death from a minimal proapoptotic region of E2F-1. Oncogene 2006; 25: 5656-5663.

35. O'Prey J, Skommer J, Wilkinson S, Ryan KM. Analysis of DRAM-related proteins reveals evolutionarily conserved and divergent roles in the control of autophagy. Cell Cycle 2009; 8: 2260-2265.

Cell Death and Disease is an open-access journal published by Nature Publishing Group. This work is licensed under the Creative Commons Attribution-Noncommercial-No Derivative Works 3.0 Unported License. To view a copy of this license, visit http://creativecommons.org/licenses/by-nc-nd/3.0/

Supplementary Information accompanies the paper on Cell Death and Disease website (http://www.nature.com/cddis) 